\title{
Sur quelques formules générales dans le calcul des opérations.
}

(Par M. Spottiswoode à Londres.)

Dans un mémoire qui vient de paraitre dans les Philosophical Transactions de Londres j'ai développé la théorie du symbole opératif

$$
y \frac{d}{d x}+x \frac{d}{d y},
$$

et j'y ai ajouté quelques formules relatives au symbole plus général:

$$
x_{i_{1}} \frac{d}{d x_{1}}+x_{i_{2}} \frac{d}{d x_{2}}+\cdots
$$

Je me propose d'indiquer dans les pages suivantes la méthode par laquelle je suis arrivé aux formules dont il s'agit.

Soit $i_{1}, i_{2}, \ldots$ une permutation quelconque de la série $1,2, \ldots$; et représentons cette permutation par l'équation symbolique

$$
i_{1}, i_{2}, \ldots=P_{i}(1,2, \ldots) \text {. }
$$

De la même manière une seconde permutation quelconque $P_{j}$ de la série $i_{1}, i_{2}, \ldots$ sera représenté par $j_{i_{1}}, j_{i_{2}}, \ldots$, ou, plus simplement, par $j i_{1}, j i_{2}, \ldots$ ce qui sera une permutation nouvelle de la série primitive 1, 2, ... Ecrivons d'une manière symbolique

ou d'une façon plus abrégée

$$
\begin{aligned}
j i_{1}, j i_{2}, \ldots & =P_{j i}(1,2, \ldots) \\
=P_{j}\left(i_{1}, i_{2}, \ldots\right) & =P_{j} P_{i}(1,2, \ldots)
\end{aligned}
$$

$$
\boldsymbol{P}_{j i}=\boldsymbol{P}_{j} \boldsymbol{P}_{i}
$$

Cette notation pourra être étendue à un nombre quelconque de permutations employées successivement.

Soient $x_{1}, x_{2}, \ldots$ des variables quelconques; et soit.

$$
\begin{aligned}
& \nabla_{i}=x_{i_{1}} \frac{d}{d x_{1}}+x_{i_{2}} \frac{d}{d x_{2}}+\cdots, \\
& \nabla_{j}=x_{j_{1}} \frac{d}{d x_{1}}+x_{j_{2}} \frac{d}{d x_{2}}+\cdots, \\
& D_{i}=x_{i_{1}} \frac{d^{\prime}}{d x_{1}}+x_{i_{2}} \frac{d^{\prime}}{d x_{2}}+\cdots, \\
& D_{j}=x_{j_{1}} \frac{d^{\prime}}{d x_{1}}+x_{j_{2}} \frac{d^{\prime}}{d x_{2}}+\cdots \text {, }
\end{aligned}
$$


Les notations $\frac{d^{\prime}}{d x_{1}}, \frac{d^{\prime}}{d x_{2}}, \ldots$ qui se trouvent dans $D_{i}, D_{j}, \ldots$ signifient que les différentiations dont il s'agit, s'exécutent bien sur les fonctions que l'on s'est abstenu d'écrire et auxquelles s'appliquent les opérations $D_{i}, D_{j}, \ldots$, mais que ces différentiations ne s'exécutent point sur les variables $x_{1}, x_{2}, \ldots$ en tant qu'elles entrent explicitement comme coefficients dans les expressions $D_{i}, D_{j}, \ldots$ Au contraire les différentiations $\frac{d}{d x_{1}}, \frac{d}{d x_{2}}, \ldots$ qui se trouvent dans $\nabla_{i}, \nabla_{j}, \ldots$ s'exécutent indistinctement. Cela posé on trouve

$$
\begin{gathered}
\boldsymbol{D}_{\boldsymbol{j}} \boldsymbol{D}_{\boldsymbol{i}}=\nabla_{j} \nabla_{\boldsymbol{i}}-\nabla_{j i} \\
\boldsymbol{D}_{k} \boldsymbol{D}_{\boldsymbol{j}} \boldsymbol{D}_{\boldsymbol{i}}=\nabla_{k} \nabla_{j} \nabla_{\boldsymbol{i}}-\nabla_{k j} \nabla_{\boldsymbol{i}}-\nabla_{j} \nabla_{k i}-\nabla_{k} \nabla_{j i}+\nabla_{k j i}+\nabla_{j k i} \\
\boldsymbol{D}_{l} \boldsymbol{D}_{k} \boldsymbol{D}_{j} \boldsymbol{D}_{i}=\nabla_{l} \nabla_{k} \nabla_{j} \nabla_{\boldsymbol{i}}-\nabla_{\boldsymbol{l}} \nabla_{j} \nabla_{\boldsymbol{i}}-\nabla_{k} \nabla_{l j} \nabla_{i}-\nabla_{k} \nabla_{j} \nabla_{l i} \\
-\nabla_{l} \nabla_{k j} \nabla_{i}-\nabla_{l} \nabla_{j} \nabla_{k i}-\nabla_{l} \nabla_{k} \nabla_{j i} \\
+\nabla_{l k j} \nabla_{i}+\nabla_{j} \nabla_{l k i}+\nabla_{k} \nabla_{l j i}+\nabla_{l} \nabla_{j k i} \\
+\nabla_{k l j} \nabla_{i}+\nabla_{j} \nabla_{k l i}+\nabla_{k} \nabla_{j l i}+\nabla_{l} \nabla_{k j i} \\
+\nabla_{k j} \nabla_{l i}+\nabla_{l j} \nabla_{k i}+\nabla_{l k} \nabla_{j i} \\
-\nabla_{l k j i}-\nabla_{k l j i}-\nabla_{k j l i}-\nabla_{l j k i}-\nabla_{j l k i}-\nabla_{j k l i}
\end{gathered}
$$

et ainsi de suite pour un nombre quelconque de $D^{\prime} s$ et de $\nabla^{\prime} s$. Il y a des cas particuliers dans lesquels ces expressions prennent une forme plus symmétrique. Si les permutations satisfont à la seconde équation du système

$$
P(j, k)=P(k, j), \quad P(k, i)=P(i, k), \quad P(i, j)=P(j, i),
$$

le groupe $\nabla_{k j}, \nabla_{k i}, \nabla_{j i}$ pourra être remplacé par le groupe $\nabla_{k j}, \nabla_{i k}, \nabla_{j i}$, dans lequel les indices se trouvent rangés selon l'ordre cyclique. Si les permutations satisfont à la première et à la troisième équation du système écrit, le groupe dont il s'agit pourra au contraire être remplacé par le groupe $\nabla_{j k}, \nabla_{h i}, \nabla_{i j}$. Si de plus les permutations satisfont à la première équation du système

$$
\begin{aligned}
& \boldsymbol{P}\{i(j, k)\}=\boldsymbol{P}\{(j, k) i\}, \\
& \boldsymbol{P}\{j(k, i)\}=\boldsymbol{P}\{(k, i) j\}, \\
& \boldsymbol{P}\{k(i, j)\}=\boldsymbol{P}\{(i, j) k\},
\end{aligned}
$$

l'expression pour $D_{k} D_{j} D_{\boldsymbol{i}}$ s'écrira de la manière suivante:

ou de celle-ci:

$$
\nabla_{k} \nabla_{j} \nabla_{i}-\nabla_{i} \nabla_{k j}-\nabla_{j} \nabla_{i k}-\nabla_{k} \nabla_{j i}+2 \nabla_{k j i}
$$

$$
\nabla_{k} \nabla_{j} \nabla_{i}-\nabla_{i} \nabla_{j k}-\nabla_{j} \dot{\nabla}_{k i}-\nabla_{k} \nabla_{i j}+\mathbf{2} \nabla_{k j i}
$$


selon que ou la seconde, ou la première et la troisième condition du premier système auront lieu. Si la seconde et la troisième condition du second système sont remplies, la même expression prend l'une ou l'autre des formes

ou

$$
\nabla_{k} \nabla_{j} \nabla_{i}-\nabla_{k j} \nabla_{i}-\nabla_{i k} \nabla_{j}-\nabla_{j i} \nabla_{k}+\mathbf{2} \nabla_{j i k}
$$

$$
\nabla_{k} \nabla_{j} \nabla_{i}-\nabla_{j k} \nabla_{i}-\nabla_{l i} \nabla_{j}-\nabla_{i j} \nabla_{k}+\mathbf{2} \nabla_{j i k}
$$

Dans le cas de $D_{l} D_{k} D_{j} D_{i}$ il serait presque nécessaire de particulariser les conditions auxquelles on assujettit les permutations.

En revenant au cas de $D_{j} D_{i}$, on établit immédiatemènt le système quadratique

$$
\begin{aligned}
\boldsymbol{D}_{i}^{2} & =\nabla_{i}^{2}-\nabla_{i^{2}}, \\
\boldsymbol{D}_{j} \boldsymbol{D}_{i} & =\nabla_{j} \nabla_{i}-\nabla_{j i}, \\
\boldsymbol{D}_{j}^{2} & =\nabla_{j}^{2}-\nabla_{j^{2}}
\end{aligned}
$$

et on en compose la rélation plus générale

dans laquelle

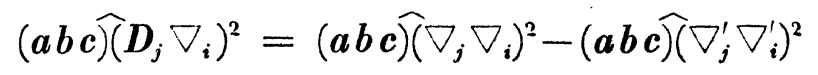

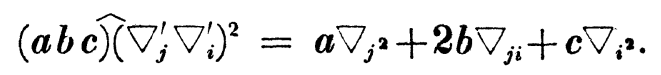

Ne perdons pas de vue d'ailleurs que dans une équation quelconque que l'on écrit les symboles $j, i$ devront toujours suivre le même ordre dans les deux parties de l'équation.

Pour le troisième degré on trouve

$$
\begin{aligned}
\boldsymbol{D}_{j}^{3} & =\nabla_{j}^{3}-\mathbf{3} \nabla_{j} \nabla_{j^{2}}+\mathbf{2} \nabla_{j^{3}} \\
\boldsymbol{D}_{j}^{2} \boldsymbol{D}_{i} & =\nabla_{j}^{2} \nabla_{i}-\nabla_{j^{2}} \nabla_{i}-\mathbf{2} \nabla_{j} \nabla_{j i}+\mathbf{2} \nabla_{j^{2}} \\
& =\nabla_{j} \nabla_{i} \nabla_{j}-\nabla_{j i} \nabla_{j}-\nabla_{i} \nabla_{j^{2}}-\nabla_{j} \nabla_{i j}+\nabla_{j i j}+\nabla_{i j^{2}} \\
& =\nabla_{i} \nabla_{j}^{2}-\nabla_{i j} \nabla_{j}-\nabla_{j} \nabla_{i j}-\nabla_{i} \nabla_{j^{2}}+\nabla_{i j^{2}}+\nabla_{j i j}
\end{aligned}
$$

d'où l'on tire le système cubique

$$
\begin{aligned}
D_{j}^{3}= & \nabla_{j}^{3}-3 \nabla_{j} \nabla_{j 2}+2 \nabla_{j 3}, \\
3 \boldsymbol{D}_{j}^{2} \boldsymbol{D}_{i}= & \nabla_{j}^{2} \nabla_{i}+\nabla_{j} \nabla_{i} \nabla_{j}+\nabla_{i} \nabla_{j}^{2} \\
& -\nabla_{j 2} \nabla_{i}-\nabla_{j i} \nabla_{j}-\nabla_{i j} \nabla_{j}-2 \nabla_{i} \nabla_{j 2}-2 \nabla_{j} \nabla_{i j}-2 \nabla_{j} \nabla_{j i} \\
& +2 \nabla_{j 2}+2 \nabla_{j i j}+2 \nabla_{i j 2}, \\
3 D_{j} D_{i}^{2}= & \nabla_{j} \nabla_{i}^{2}+\nabla_{i} \nabla_{j} \nabla_{i}+\nabla_{i}^{2} \nabla_{j} \\
& -\nabla_{i 2} \nabla_{j}-\nabla_{i j} \nabla_{i}-\nabla_{j i} \nabla_{i}-2 \nabla_{j} \nabla_{i 2}-2 \nabla_{i} \nabla_{j i}-2 \nabla_{i} \nabla_{i j} \\
& +2 \nabla_{j i 2}+2 \nabla_{i j i}+2 \nabla_{i 2 j}, \\
D_{i}^{3}= & \nabla_{i}^{3}-3 \nabla_{i} \nabla_{i 2}+2 \nabla_{i 2} .
\end{aligned}
$$

Journal für Mathematik Bd. LIX. Heft 4. 
Or

$$
\begin{aligned}
& \left(\boldsymbol{a b c} \widehat{c} \nabla_{j} \nabla_{i}\right)^{2}=\boldsymbol{a} \nabla_{j}^{2}+\boldsymbol{b}\left(\nabla_{j} \bar{\nabla}_{i}+\nabla_{i} \nabla_{j}\right)+\boldsymbol{c} \nabla_{i}^{2} \\
& \left(a b c d \widehat{)} \nabla_{j} \nabla_{i}\right)^{3} \\
& =\boldsymbol{a} \nabla_{j}^{3}+\boldsymbol{b}\left(\nabla_{j}^{2} \nabla_{i}+\nabla_{j} \nabla_{i} \nabla_{j}+\nabla_{i} \nabla_{j}^{2}\right)+\boldsymbol{c}\left(\nabla_{j} \nabla_{i}^{2}+\nabla_{i} \nabla_{j} \nabla_{i}+\nabla_{i}^{2} \nabla_{j}\right)+\boldsymbol{d} \nabla_{i}^{3}, \\
& \left(\boldsymbol{a b c d} \widehat{\left(\nabla_{j}\right.} \nabla_{i} \widehat{)} \nabla_{j}^{\prime} \nabla_{i}^{\prime}\right)^{2} \\
& =\boldsymbol{a} \nabla_{j} \nabla_{\boldsymbol{j} \mathbf{2}}+\boldsymbol{b}\left(\nabla_{i} \nabla_{j i}+\nabla_{j} \nabla_{i j}+\nabla_{i} \nabla_{\boldsymbol{j} \mathbf{2}}\right)+\boldsymbol{c}\left(\nabla_{i} \nabla_{j i}+\nabla_{i} \nabla_{i j}+\nabla_{j} \nabla_{i \mathbf{2}}\right)+\boldsymbol{d} \nabla_{i} \nabla_{i \mathbf{2}}, \\
& \left(\boldsymbol{a b c d} \widehat{\left(\nabla_{j}^{\prime}\right.} \nabla_{i}^{\prime} \widehat{)}^{2}\left(\nabla_{j} \nabla_{i}\right)\right. \\
& =\boldsymbol{a} \nabla_{j} \nabla_{j}+\boldsymbol{b}\left(\nabla_{j i} \nabla_{j}+\nabla_{i j} \nabla_{j}+\nabla_{j \mathbf{2}} \nabla_{i}\right)+\boldsymbol{c}\left(\nabla_{j i} \nabla_{i}+\nabla_{i j} \nabla_{i}+\nabla_{i \mathbf{2}} \nabla_{j}\right)+\boldsymbol{d} \nabla_{i \mathbf{2}} \nabla_{i}, \\
& (a b c d)\left(\nabla_{j}^{\prime} \nabla_{i}^{\prime}\right)^{3} \\
& =\boldsymbol{a} \nabla_{\boldsymbol{j} 3}+\boldsymbol{b}\left(\nabla_{\boldsymbol{j} \mathbf{2}} \nabla_{j i j}+\nabla_{i j^{2}}\right)+\boldsymbol{c}\left(\nabla_{j \boldsymbol{i}^{2}}+\nabla_{i j i}+\nabla_{i 2 j}\right)+\boldsymbol{d} \nabla_{i 3}
\end{aligned}
$$

d'où

$$
\begin{aligned}
& \left(a b c d \widehat{)}\left(D_{j} D_{i}\right)^{3}\right.
\end{aligned}
$$

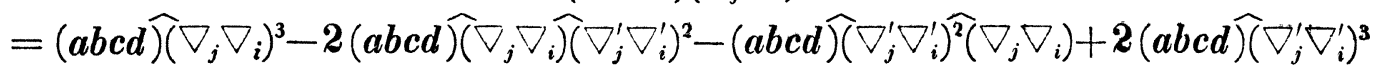
équation que l'on peut écrire symboliquement ainsi:

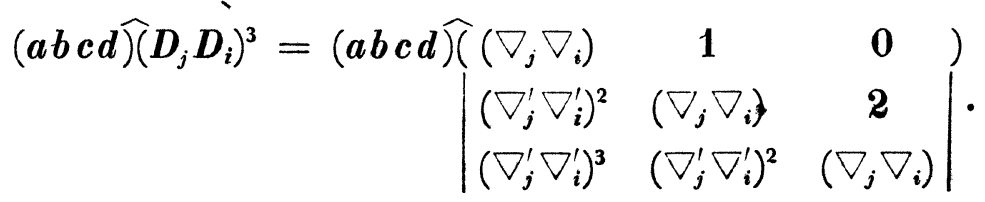

En se bornant au cas de deux facteurs opératifs, on a les formules suivantes:

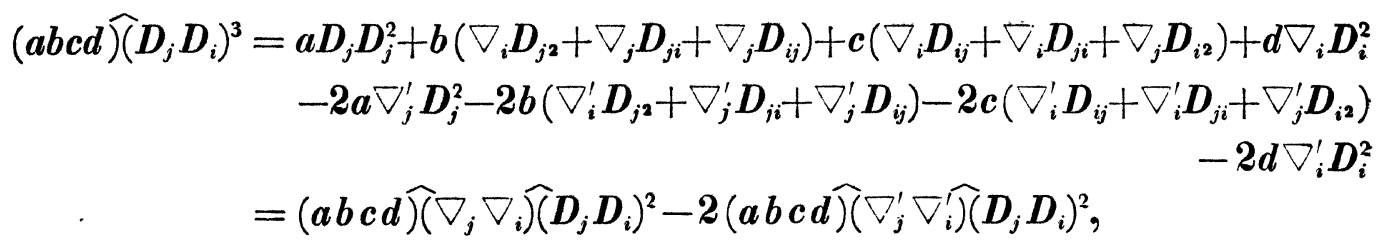

de même:

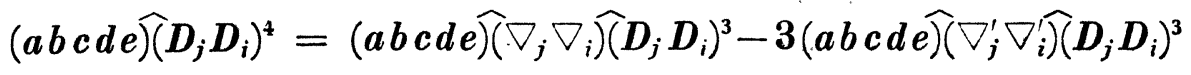

et pour le $n^{\text {ième }}$ degré :

$$
\left(a b \ldots \widehat{)}\left(D_{j} D_{i}\right)^{n}=(a b \ldots)\left(\nabla_{j} \nabla_{i} \widehat{)}\left(D_{j} D_{i}\right)^{n-1}-(n-1)(a b \ldots)\left(\nabla_{j}^{\prime} \nabla_{i} \widehat{)}\left(D_{j} D_{i}\right)^{n-1}\right.\right.\right.
$$

ou

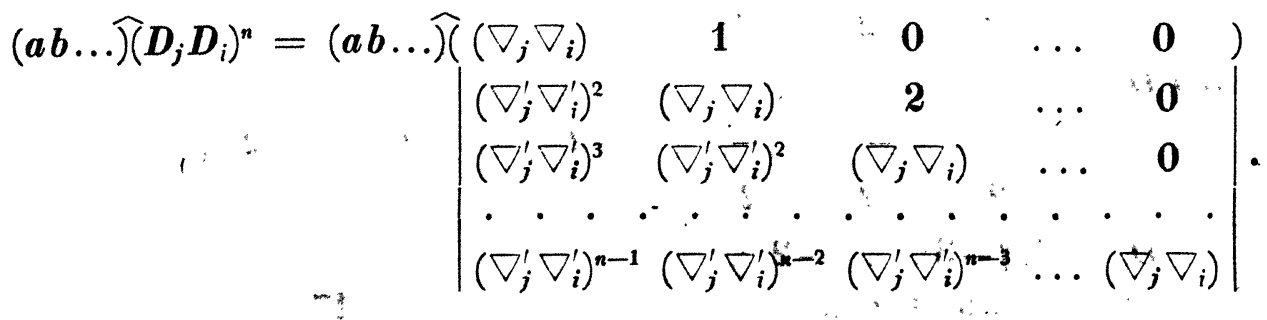


Pour montrer que la même loi a lieu dans le cas de plusieurs facteurs opératifs, introduisons un troisième facteur $D_{k}$; et, pour abréger les formules, nous n'écrivons que les indices, de sorte que dans les calculs suivants on écrira $i j k$ au lieu de $\nabla_{i} \nabla_{j} \nabla_{k} ; i^{\prime} j^{\prime} k$ au lieu de $\nabla_{i}^{\prime} \nabla_{j}^{\prime} \nabla_{k}$ ou $\nabla_{i j} \nabla_{k}$; et ainsi de suite. Cela posé on aura

$$
\begin{aligned}
D_{k} D_{j} D_{i} & =k j i-k^{\prime} j^{\prime} i-j k^{\prime} i^{\prime}-k j^{\prime} i^{\prime}+k^{\prime} j^{\prime} i^{\prime}+j^{\prime} k^{\prime} i^{\prime} \\
& =j k i-j^{\prime} k^{\prime} i-k j^{\prime} i^{\prime}-j k^{\prime} i^{\prime}+j^{\prime} k^{\prime} i^{\prime}+k^{\prime} j^{\prime} i^{\prime} \\
& =i j k-i^{\prime} j^{\prime} k-j i^{\prime} k^{\prime}-i j^{\prime} k^{\prime}+i^{\prime} j^{\prime} k^{\prime}+j^{\prime} i^{\prime} k^{\prime} \\
& =k i j-k^{\prime} i^{\prime} j-i k^{\prime} j^{\prime}-k i^{\prime} j^{\prime}+k^{\prime} i^{\prime} j^{\prime}+i^{\prime} k^{\prime} j^{\prime} \\
& =i k j-i^{\prime} k^{\prime} j-k i^{\prime} j^{\prime}-i k^{\prime} j^{\prime}+i^{\prime} k^{\prime} j^{\prime}+k^{\prime} i^{\prime} j^{\prime} \\
& =j i k-j^{\prime} i^{\prime} k-i j^{\prime} k^{\prime}-j i^{\prime} k^{\prime}+j^{\prime} i^{\prime} k^{\prime}+i^{\prime} j^{\prime} k^{\prime}
\end{aligned}
$$

d'ou l'on tire le système cubique

$$
\begin{aligned}
6 D_{k} D_{j} D_{i}= & k j i+j k i+i j k+k i j+i k j+j i k \\
& -k^{\prime} j^{\prime} i-j^{\prime} k^{\prime} i-2 i k^{\prime} j^{\prime}-2 i j^{\prime} k^{\prime} \\
& -i^{\prime} k^{\prime} j-k^{\prime} i^{\prime} j-2 j i^{\prime} k^{\prime}-2 j k^{\prime} i^{\prime} \\
& -j^{\prime} i^{\prime} k-i^{\prime} j^{\prime} k-2 k j^{\prime} i^{\prime}-2 k i^{\prime} j^{\prime} \\
& +2\left(k^{\prime} j^{\prime} i^{\prime}+j^{\prime} k^{\prime} i^{\prime}+i^{\prime} j^{\prime} k^{\prime}+k^{\prime} i^{\prime} j^{\prime}+i^{\prime} k^{\prime} j^{\prime}+j^{\prime} i^{\prime} k^{\prime}\right), \\
3 D_{k}^{2} D_{j}= & k^{2} j+k j k+j k^{2}+2\left(j^{\prime 2} k^{\prime}+j^{\prime} k^{\prime} j^{\prime}+k^{\prime} j^{\prime 2}\right) \\
& -k^{\prime 2} j-k^{\prime} j^{\prime} k-j^{\prime} k^{\prime} j-2\left(j k^{\prime 2}+k j^{\prime} k^{\prime}+k k^{\prime} j^{\prime}\right), \\
3 D_{k} D_{j}^{2}= & k j^{2}+j k j+j^{2} k+2\left(k^{\prime} j^{\prime 2}+j^{\prime} k^{\prime} j^{\prime}+j^{\prime 2} k^{\prime}\right) \\
& -j^{\prime 2} k-j^{\prime} k^{\prime} j-k^{\prime} j^{\prime} j-2\left(k j^{\prime 2}+j k^{\prime} j^{\prime}+j j^{\prime} k^{\prime}\right), \\
3 D_{i}^{2} D_{k}= & i^{2} k+i k i+k i^{2}+2\left(i^{\prime 2} k^{\prime}+i^{\prime} k^{\prime} i^{\prime}+k^{\prime} i^{\prime 2}\right) \\
& -i^{\prime 2} k-i^{\prime} k^{\prime} i-k^{\prime} i^{\prime} i-2\left(k i^{\prime 2}+i k^{\prime} i^{\prime}+i i^{\prime} k^{\prime}\right), \\
3 D_{i} D_{k}^{2}= & i k^{2}+k i k+k^{2} i+2\left(i^{\prime} k^{\prime 2}+k^{\prime} i^{\prime} k^{\prime}+k^{\prime 2} i^{\prime}\right) \\
& -k^{\prime 2} i-k^{\prime} i^{\prime} k-i^{\prime} k^{\prime} k-2\left(i k^{\prime 2}+k i^{\prime} k^{\prime}+k k^{\prime} i^{\prime}\right), \\
3 D_{j}^{2} D_{i}= & j^{2} i+j i j+i j^{2}+2\left(j^{\prime 2} i^{\prime}+j^{\prime} i^{\prime} j^{\prime}+i^{\prime} j^{\prime 2}\right) \\
& -j^{\prime 2} i-j^{\prime} i^{\prime} j-i^{\prime} j^{\prime} j-2\left(i j^{\prime 2}+j i^{\prime} j^{\prime}+j j^{\prime} i^{\prime}\right), \\
3 D_{j} D_{i}^{2}= & j i^{2}+i j i-i^{2} j+2\left(j^{\prime} i^{\prime 2}+i^{\prime} j^{\prime} i^{\prime}+i^{\prime 2} j^{\prime}\right) \\
& -i^{\prime 2} j-i^{\prime} j^{\prime} i-j^{\prime} i^{\prime} i-2\left(j i^{\prime 2}+i j^{\prime} i^{\prime}+i i^{\prime} j^{\prime}\right), \\
D_{k}^{3}= & k^{3}-3 k k^{\prime 2}+2 k^{\prime 3}, \\
D_{j}^{3}= & j^{3}-3 j j^{\prime 2}+2 j^{\prime 3}, \\
D_{i}^{3}= & i^{3}-3 i i^{\prime 2}+2 i^{\prime 3} .
\end{aligned}
$$


Or

d'où

$$
\begin{aligned}
& \left(a b c f f _ { 1 } g g _ { 1 } h h _ { 1 } e \widehat { e } \left(i j k \widehat{)\left(i^{\prime} j^{\prime} k^{\prime}\right)^{2}}\right.\right. \\
& =a k k^{\prime 2}+b j j^{\prime 2}+c i i^{\prime 2} \\
& +f\left(j i^{\prime 2}+i j^{\prime} i^{\prime}+i i^{\prime} j^{\prime}\right)+f_{1}\left(i j^{2}+j i^{\prime} j^{\prime}+j j^{\prime} i^{\prime}\right) \\
& +g\left(i k^{\prime 2}+k i^{\prime} k^{\prime}+k k^{\prime} i^{\prime}\right)+g_{1}\left(k i^{\prime 2}+i k^{\prime} i^{\prime}+i i^{\prime} k^{\prime}\right) \\
& +h\left(k j^{\prime 2}+j k^{\prime} j^{\prime}+j j^{\prime} k^{\prime}\right)+h_{1}\left(i k^{\prime 2}+k j^{\prime} k^{\prime}+k k^{\prime} j^{\prime}\right) \\
& +e\left(i j^{\prime} k^{\prime}+i k^{\prime} j^{\prime}+j k^{\prime} i^{\prime}+j i^{\prime} k^{\prime}+k i^{\prime} j^{\prime}+k j^{\prime} i^{\prime}\right)
\end{aligned}
$$

$$
\begin{aligned}
& \left(a b c f f_{1} g g_{1} h h_{1} e \widehat{\left(D_{k} D_{j} D_{i}\right)^{3}}\right. \\
& =\left(a b \ldots \widehat{)} \nabla_{k} \nabla_{j} \nabla_{i}\right)^{3}-\mathbf{2}\left(a b \ldots \widehat{)} \nabla_{k} \nabla_{j} \nabla_{i} \widehat{)(} \nabla_{k}^{\prime} \nabla_{j}^{\prime} \nabla_{i}^{\prime}\right)^{2} \\
& -\left(\boldsymbol{a} b . . . \widehat{(} \nabla_{k}^{\prime} \nabla_{j}^{\prime} \nabla_{i}^{\prime} \widehat{2}^{2} \nabla_{k} \nabla_{j} \nabla_{i}\right)+2\left(a b \ldots \widehat{)} \nabla_{k}^{\prime} \nabla_{j}^{\prime} \nabla_{i}^{\prime}\right)^{3}
\end{aligned}
$$

et comme les formules, que nous avons établiés dans le cas de deux facteurs opératifs ${ }^{*} D_{j} D_{i}$, peuvent s'étendre au cas de trois, on conclut qu'en général on a la formule

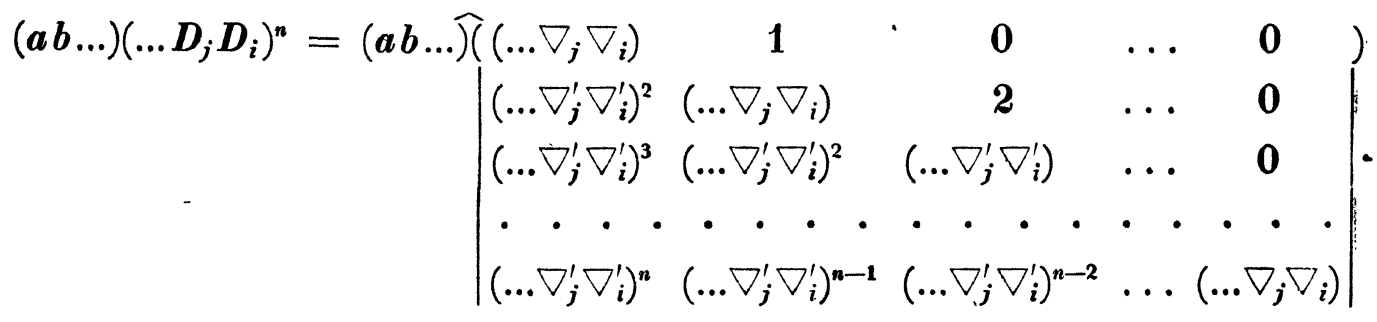

Pour calculer l'effet des opérations $\nabla_{i}$ sur une fonction donnée, soit proposée la suivante

$$
u=\Sigma\left(1^{\alpha_{1}} 2^{\alpha_{2}} \ldots\right) x_{1}^{\alpha_{1}} x_{2}^{\alpha_{2}} \ldots
$$

dans laquelle $\left(1^{\alpha_{1}} 2^{\alpha_{2}} \ldots\right)$ représente une seule quantité, et où l'on a

Cela posé on trouve

$$
\alpha_{1}+\alpha_{2}+\cdots=n \text {. }
$$

$$
\begin{aligned}
\nabla_{i} u= & \Sigma\left(1^{\alpha_{1}} 2^{\alpha_{2}} \ldots\right)\left\{\alpha_{1} x_{1}^{\alpha_{1}-1} x_{2}^{\alpha_{2}} \ldots x_{i_{1}}^{\alpha_{i_{1}}+1} \ldots\right. \\
& +\alpha_{2} x_{1}^{\alpha_{1}} x_{2}^{\alpha_{2}-1} \ldots x_{i_{2}}^{\alpha_{i_{2}}+1} \ldots \\
& +\cdots \\
= & \Sigma\left\{\left(\alpha_{1}+1\right)\left(1^{\alpha_{1}+1} 2^{\alpha_{2}} \ldots i_{1}^{\alpha_{i_{1}}-1} \ldots\right)\right. \\
& +\left(\alpha_{2}+1\right)\left(1^{\alpha_{1}} 2^{\alpha_{2}+1} \ldots i_{2}^{\alpha_{i_{2}}-1} \ldots\right) \\
& +\cdots \\
= & \Sigma\left\{\left(\alpha_{1}+1\right) e^{\frac{d}{d \alpha_{1}}-\frac{d}{d \alpha_{i_{1}}}}+\left(\alpha_{2}+1\right) e^{\frac{d}{d \alpha_{2}}-\frac{d}{d \alpha_{i_{1}}}}+\ldots\right\}\left(x^{\alpha_{1}} 2^{\alpha_{2}} \ldots\right) x_{1}^{\alpha_{1}} x_{2}^{\alpha_{2}} \ldots
\end{aligned}
$$


De la même manière on s'élève aux formules plus générales

$$
\nabla_{i}^{p} u=\Sigma\left\{\Sigma\left(\alpha_{r}+1\right) e^{\frac{d}{d \alpha_{r}}-\frac{d}{d \alpha_{i_{r}}}}\right\}^{p}\left(1^{\alpha_{1}} 2^{\alpha_{2}} \ldots\right) x_{1}^{\alpha_{1}} x_{2}^{\alpha_{2}} \ldots
$$

et

$F\left(\ldots \nabla_{j} \nabla_{i}\right) u=\Sigma F\left\{\ldots, \Sigma\left(\alpha_{s}+1\right) e^{\frac{d}{d \alpha_{s}}-\frac{d}{d \alpha_{j_{s}}}}, \Sigma\left(\alpha_{r}+1\right) e^{\frac{d}{d \alpha_{r}}-\frac{d}{d \alpha_{i_{r}}}}\right\}\left(1^{\alpha_{1}} 2^{\alpha_{2}} \ldots\right) x_{1}^{\alpha_{1}} x_{2}^{\alpha_{2}} \ldots$ où il est toujours essentiel de conserver inaltéré l'ordre des multiplications.

Considérons le cas où les symboles de simple différentiation sont multipliés par des fonctions linéares des variables. Soient

$$
\begin{gathered}
u_{11}=\alpha_{11} x_{1}+\beta_{11} x_{2}+\cdots, \quad u_{12}=\alpha_{12} x_{1}+\beta_{12} x_{2}+\cdots, \ldots \\
u_{21}=\alpha_{21} x_{1}+\beta_{21} x_{2}+\cdots, \quad u_{22}=\alpha_{22} x_{1}+\beta_{22} x_{2}+\cdots, \ldots \\
\ldots \ldots \ldots \\
\nabla_{1}=u_{11} \frac{d}{d x_{1}}+u_{12} \frac{d}{d x_{2}}+\cdots \\
\nabla_{2}=u_{21} \frac{d}{d x_{1}}+u_{22} \frac{d}{d x_{2}}+\cdots
\end{gathered}
$$

Alors

$$
\begin{gathered}
D_{2} D_{1}=\nabla_{2} \nabla_{1}-\left\{\left(\alpha_{11} \alpha_{21}+\beta_{11} \alpha_{22}+\cdots\right) x_{1}+\left(\alpha_{11} \beta_{21}+\beta_{11} \beta_{22}+\cdots\right) x_{2}+\cdots\right\} \frac{d}{d x_{1}} \\
-\left\{\left(\alpha_{12} \alpha_{21}+\left(\beta_{12} \alpha_{22}+\cdots\right) x_{1}+\left(\alpha_{12} \beta_{21}+\beta_{12} \beta_{22}+\cdots\right) x_{2}+\cdots\right\} \frac{d}{d x_{2}}\right. \\
-\cdots
\end{gathered}
$$

Or, les coefficients des $x$ sont tous compris dans le produit

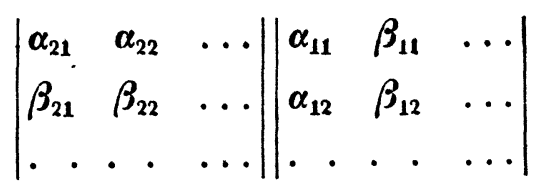

et les termes de chaque ligne et de chaque colonne correspondent de sorte qu'en posant

$$
\left(\begin{array}{ccc}
a & b & \ldots \\
a_{1} & b_{1} & \ldots \\
\ldots & \ldots & \ldots
\end{array} \mid \widehat{\left(x x_{1} \ldots\right)\left(y y_{1} \ldots\right)=\left(a x+b x_{1}+\cdots\right) y} \begin{array}{c}
+\left(a_{1} x+b_{1} x_{1}+\cdots\right) y_{1} \\
+\cdots
\end{array}\right.
$$


on peut écrire

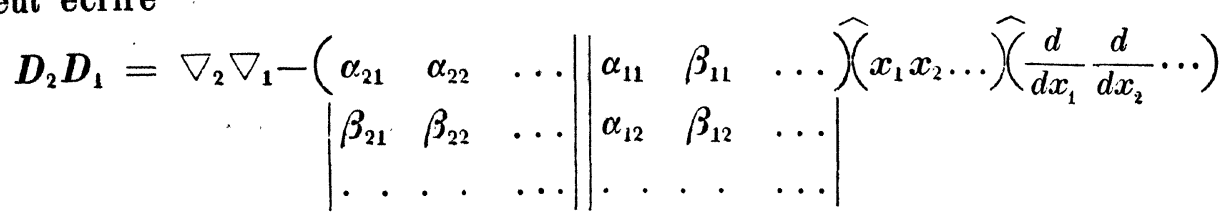

De la même manière on trouve

$$
\begin{aligned}
& D_{3} D_{2} D_{1} \rightleftharpoons \nabla_{3} \nabla_{2} \nabla_{1}-\left(\begin{array}{lll}
\alpha_{31} & \alpha_{32} & \ldots \|
\end{array} \mid \begin{array}{lll}
\alpha_{21} & \beta_{21} & \ldots
\end{array}\right) \widehat{\left(x_{1} x_{2} \ldots\right)}\left(\frac{d}{d x_{1}} \frac{d}{d x_{2}} \ldots\right) \nabla_{1}
\end{aligned}
$$

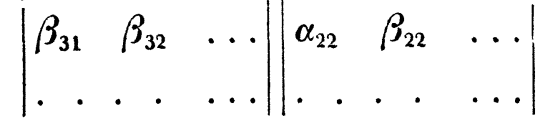

$$
\begin{aligned}
& -\nabla_{2}\left(\begin{array}{lll}
\alpha_{31} & \alpha_{32} & \ldots
\end{array} \| \begin{array}{llll}
\alpha_{11} & \beta_{11} & \ldots
\end{array}\right)\left(x_{1} x_{2} \ldots\right) \widehat{\left(\frac{d}{d x_{1}} \frac{d}{d x_{2}} \ldots\right)}
\end{aligned}
$$

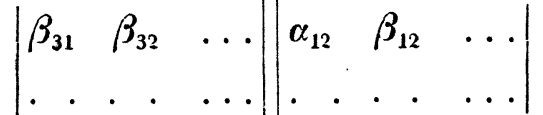

$$
\begin{aligned}
& -\nabla_{3}\left(\begin{array}{lll}
\alpha_{21} & \alpha_{22} & \ldots
\end{array} \cdot \begin{array}{lll}
\alpha_{11} & \beta_{11} & \ldots
\end{array}\right)\left(x_{1} x_{2} \ldots\right) \widehat{\left(\frac{d}{d x_{1}} \frac{d}{d x_{2}} \ldots\right)}
\end{aligned}
$$

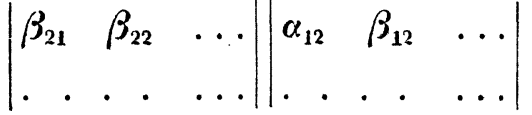

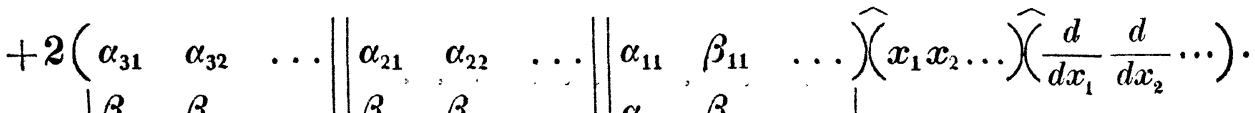

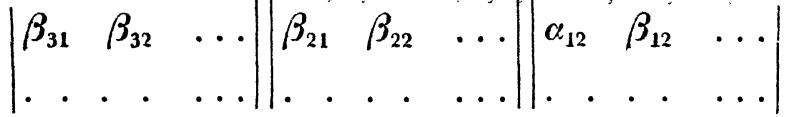

En se servant d'une notation symbolique dont la signification est manifeste, on peut mettre cette expression sous la forme

$$
\nabla_{3} \nabla_{2} \nabla_{1}-\nabla_{3}^{\prime} \nabla_{2}^{\prime} \nabla_{1}-\nabla_{2} \nabla_{3}^{\prime} \nabla_{1}^{\prime}-\nabla_{3} \nabla_{2}^{\prime} \nabla_{1}^{\prime}+2 \nabla_{3}^{\prime} \nabla_{2}^{\prime} \nabla_{1}^{\prime}
$$

Rélativement aux produits symboliques $\nabla_{3}^{\prime} \nabla_{2}^{\prime}, \nabla_{3}^{\prime} \nabla_{1}^{\prime}, \nabla_{2}^{\prime} \nabla_{1}^{\prime}, \nabla_{3}^{\prime} \nabla_{2}^{\prime} \nabla_{1}^{\prime}$, il est essentiel de se souvenir que. quand on forme les produits de déterminants tels que le suivant

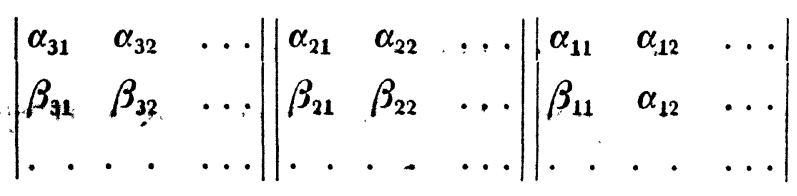

les lignes veriticales doivent remplacer les lignes horizontales et vice versa dans le derniar dèterminant de chaque produit. De plus, si l'on convient de remplacer dans le développement des produits chaque terme de la forme 
$\nabla_{3}^{\prime} \nabla_{2} \nabla_{1}^{\prime}$ par le suivant $\nabla_{2} \nabla_{3}^{\prime} \nabla_{1}^{\prime}$, on peut écrire

$$
D_{3} D_{2} D_{1}=\left|\begin{array}{ccc}
\nabla_{3} & \nabla_{2}^{\prime} & \nabla_{1}^{\prime} \\
\nabla_{3}^{\prime} & \nabla_{2} & \nabla_{1}^{\prime} \\
\nabla_{3}^{\prime} & \nabla_{2}^{\prime} & \nabla_{1}
\end{array}\right| \text {. }
$$

Cette dernière formule peut aussi être déduite de celle pour $D_{2} D_{1}$ et de la considération que $\left(D_{3}^{\prime}, D_{2}^{\prime}, D_{1}^{\prime}\right.$, étant des symboles analogues à $\left.\nabla_{3}^{\prime}, \nabla_{2}^{\prime}, \nabla_{1}^{\prime}\right)$ on a

$$
\begin{aligned}
\boldsymbol{D}_{3} \boldsymbol{D}_{2} \boldsymbol{D}_{1} & =\nabla_{3} \boldsymbol{D}_{2} \boldsymbol{D}_{\mathbf{1}}-\boldsymbol{D}_{3}^{\prime} \boldsymbol{D}_{2}^{\prime} \boldsymbol{D}_{\mathbf{1}}-\boldsymbol{D}_{3}^{\prime} \boldsymbol{D}_{2} \boldsymbol{D}_{1}^{\prime} \\
& =\nabla_{3}\left(\nabla_{2} \nabla_{1}-\nabla_{2}^{\prime} \nabla_{1}^{\prime}\right)-\nabla_{\mathbf{3}}\left(\nabla_{2}^{\prime} \nabla_{1}-\nabla_{2}^{\prime} \nabla_{1}^{\prime}\right)-\nabla_{3}^{\prime}\left(\nabla_{2} \nabla_{1}^{\prime}-\nabla_{2}^{\prime} \nabla_{1}^{\prime}\right) .
\end{aligned}
$$

De la même manière on trouve aussi

$$
\begin{aligned}
& \boldsymbol{D}_{4} \boldsymbol{D}_{3} \boldsymbol{D}_{2} \boldsymbol{D}_{1}=\nabla_{4} \boldsymbol{D}_{3} \boldsymbol{D}_{2} \boldsymbol{D}_{1}-\boldsymbol{D}_{4}^{\prime} \boldsymbol{D}_{3}^{\prime} \boldsymbol{D}_{2} \boldsymbol{D}_{1}-\boldsymbol{D}_{4}^{\prime} \boldsymbol{D}_{3} \boldsymbol{D}_{2}^{\prime} \boldsymbol{D}_{1}-\boldsymbol{D}_{4}^{\prime} \boldsymbol{D}_{3} \boldsymbol{D}_{2} \boldsymbol{D}_{1}^{\prime} \\
= & \nabla_{4}\left|\begin{array}{ccc}
\nabla_{3} & \nabla_{2}^{\prime} & \nabla_{1}^{\prime} \\
\nabla_{3}^{\prime} & \nabla_{2} & \nabla_{1}^{\prime} \\
\nabla_{3}^{\prime} & \nabla_{2}^{\prime} & \nabla_{1}
\end{array}\right|-\nabla_{4}^{\prime}\left|\begin{array}{lll}
\nabla_{3}^{\prime} & \nabla_{2}^{\prime} & \nabla_{1}^{\prime} \\
\nabla_{3}^{\prime} & \nabla_{2} & \nabla_{1}^{\prime} \\
\nabla_{3}^{\prime} & \nabla_{2}^{\prime} & \nabla_{1}
\end{array}\right|-\nabla_{4}^{\prime}\left|\begin{array}{lll}
\nabla_{3} & \nabla_{2}^{\prime} & \nabla_{1}^{\prime} \\
\nabla_{3}^{\prime} & \nabla_{2}^{\prime} & \nabla_{1}^{\prime} \\
\nabla_{3}^{\prime} & \nabla_{2}^{\prime} & \nabla_{1}
\end{array}\right| \begin{array}{lll}
\nabla_{3} & \nabla_{2}^{\prime} & \nabla_{1}^{\prime} \\
\nabla_{3}^{\prime} & \nabla_{2} & \nabla_{1}^{\prime} \\
\nabla_{3}^{\prime} & \nabla_{2}^{\prime} & \nabla_{1}^{\prime}
\end{array} \mid \\
= & \left|\begin{array}{llll}
\nabla_{4} & \nabla_{3}^{\prime} & \nabla_{2}^{\prime} & \nabla_{1}^{\prime} \\
\nabla_{4}^{\prime} & \nabla_{3} & \nabla_{2}^{\prime} & \nabla_{1}^{\prime} \\
\nabla_{4}^{\prime} & \nabla_{3}^{\prime} & \nabla_{2} & \nabla_{1}^{\prime} \\
\nabla_{4}^{\prime} & \nabla_{3}^{\prime} & \nabla_{2}^{\prime} & \nabla_{1}
\end{array}\right|
\end{aligned}
$$

et en général

$$
\begin{aligned}
& D_{n} D_{n-1} \ldots D_{2} D_{1} \\
& =\nabla_{n} D_{n-1} \ldots D_{2} D_{1}-D_{n}^{\prime} D_{n-1}^{\prime} \ldots D_{2} D_{1}-\ldots-D_{n}^{\prime} D_{n-1} \ldots D_{2}^{\prime} D_{1}-D_{n}^{\prime} D_{n-1} \ldots D_{2} D_{k}^{\prime}
\end{aligned}
$$

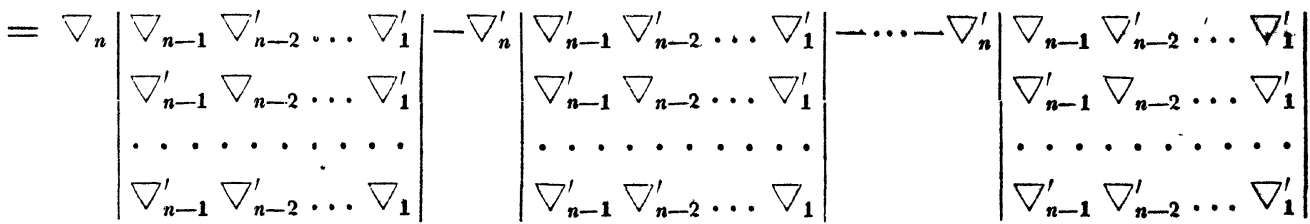

$$
\begin{aligned}
& =\left|\begin{array}{cccc}
\nabla_{n} & \nabla_{n-1}^{\prime} & \ldots & \nabla_{1}^{\prime} \\
\nabla_{n}^{\prime} & \nabla_{n-1}^{\prime} & \ldots & \nabla_{1}^{\prime} \\
\cdots & \cdots & \cdots & \cdots \\
\nabla_{n}^{\prime} & \nabla_{n-1}^{\prime} & \ldots & \nabla_{1}
\end{array}\right|
\end{aligned}
$$

On peut aussi comme dans le cas de deux variables déterminer les expressions pour les opérations de la forme

$$
s_{1} s_{2} \ldots s_{m} \frac{d^{m}}{d x^{p} d y^{q} \ldots}
$$


où

$$
\begin{aligned}
s & =\alpha x+\beta y+\cdots, \\
m & =p+q+\cdots
\end{aligned}
$$

c. à d. les calculer en termes de $s_{i} \frac{d}{d x}, s_{i} \frac{d}{d y}, \ldots$ Mais, en égard à mon mémoire ci-dessus cité, il suffira de rappeler les formules sans les démontrer. Dans le cas de trois variables et de trois fonctions $s$ on a:

$$
\begin{gathered}
6 s s_{1} s_{2} \frac{d^{3}}{d x d y d z} \\
=s \frac{d}{d x}\left(s_{1} \frac{d}{d y}-\beta_{1}\right)\left(s_{2} \frac{d}{d z}-2 \gamma_{2}\right)+s \frac{d}{d x}\left(s_{1} \frac{d}{d z}-\gamma_{1}\right)\left(s_{2} \frac{d}{d y}-2 \beta_{2}\right) \\
+s \frac{d}{d y}\left(s_{1} \frac{d}{d z}-\gamma_{1}\right)\left(s_{2} \frac{d}{d x}-2 \alpha_{2}\right)+s \frac{d}{d y}\left(s_{1} \frac{d}{d x}-\alpha_{1}\right)\left(s_{2} \frac{d}{d z}-2 \gamma_{2}\right) \\
+s \frac{d}{d z}\left(s_{1} \frac{d}{d x}-\alpha_{1}\right)\left(s_{2} \frac{d}{d y}-2 \beta_{2}\right)+s \frac{d}{d z}\left(s_{1} \frac{d}{d y}-\beta_{1}\right)\left(s_{2} \frac{d}{d x}-2 \alpha_{2}\right)
\end{gathered}
$$

ou bien

$$
\begin{aligned}
& =\left(s \frac{d}{d x}-2 \alpha\right)\left(s_{1} \frac{d}{d y}-\beta_{1}\right) s_{2} \frac{d}{d z}+\left(s \frac{d}{d x}-2 \alpha\right)\left(s_{2} \frac{d}{d y}-\beta_{2}\right) s_{1} \frac{d}{d z} \\
& +\left(s_{1} \frac{d}{d x}-2 \alpha_{1}\right)\left(s_{2} \frac{d}{d y}-\beta_{2}\right) s \frac{d}{d z}+\left(s_{1} \frac{d}{d x}-2 \alpha_{1}\right)\left(s \frac{d}{d y}-\beta\right) s_{2} \frac{d}{d z} \\
& +\left(s_{2} \frac{d}{d x}-2 \alpha_{2}\right)\left(s \frac{d}{d y}-\beta\right) s_{1} \frac{d}{d z}+\left(s_{2} \frac{d}{d x}-2 \alpha_{2}\right)\left(s_{1} \frac{d}{d y}-\beta_{1}\right) s \frac{d}{d z} .
\end{aligned}
$$

Lorsque $s, s_{1}, s_{2}$ ne sont plus des fonctions linéaires mais des fonctions quelconques de $x, y, z$ on a la formule plus générale

$$
\begin{gathered}
6 s s_{1} s_{2} \frac{d^{3}}{d x d y d z} \\
=\left(s \frac{d}{d x}-2 \frac{d s}{d x}\right)\left(s_{1} \frac{d}{d y}-\frac{d s_{1}}{d y}\right) s_{2} \frac{d}{d z}+\left(s \frac{d}{d x}-2 \frac{d s}{d x}\right)\left(s_{2} \frac{d}{d y}-\frac{d s_{2}}{d y}\right) s_{1} \frac{d}{d z} \\
+\left(s_{1} \frac{d}{d x}-2 \frac{d s_{1}}{d x}\right)\left(s_{2} \frac{d}{d y}-\frac{d s_{2}}{d y}\right) s \frac{d}{d z}+\left(s_{1} \frac{d}{d x}-2 \frac{d s_{1}}{d x}\right)\left(s \frac{d}{d y}-\frac{d s}{d y}\right) s_{2} \frac{d}{d z} \\
+\left(s_{2} \frac{d}{d x}-2 \frac{d s_{2}}{d x}\right)\left(s \frac{d}{d y}-\frac{d s}{d y}\right) s_{1} \frac{d}{d z}+\left(s_{2} \frac{d}{d x}-2 \frac{d s_{2}}{d x}\right)\left(s_{1} \frac{d}{d y}-\frac{d s_{1}}{d y}\right) s \frac{d}{d z}
\end{gathered}
$$

Je prends pour exemple de la théorie précédente le cas de trois variables $x, y, s$. 
Soit

$$
\begin{gathered}
\boldsymbol{P}=x, y, z, \\
\boldsymbol{P}_{1}=x, z, y, \\
\boldsymbol{P}_{2}=z, y, x \\
\boldsymbol{P}_{3}=y, x, z \\
\boldsymbol{P}_{4}=y, z, x \\
\boldsymbol{P}_{5}=z, x, y \\
\nabla=x \frac{d}{d x}+y \frac{d}{d y}+z \frac{d}{d z}, \\
\nabla_{1}=x \frac{d}{d x}+z \frac{d}{d y}+y \frac{d}{d z}, \\
\nabla_{2}=z \frac{d}{d x}+y \frac{d}{d y}+x \frac{d}{d z}, \\
\nabla_{3}=y \frac{d}{d x}+x \frac{d}{d y}+z \frac{d}{d z}, \\
\nabla_{4}=y \frac{d}{d x}+z \frac{d}{d y}+x \frac{d}{d z}, \\
\nabla_{5}=z \frac{d}{d x}+x \frac{d}{d y}+y \frac{d}{d z} .
\end{gathered}
$$

Au lieu de $\boldsymbol{P}_{i}$, symbole de l'une quelconque des permutations, j'écris, pour abréger, le seul indice $i$, alors la composition des permutations s'exprimera par les équations symboliques:

$$
\begin{aligned}
& 1^{2}=0 \quad 2^{2}=0 \quad 3^{2}=0 \quad 4^{2}=5 \quad 5^{2}=4 \\
& 23=4 \quad 31=4 \quad 12=4 \\
& 32=5 \quad 13=5 \quad 21=5 \\
& 14=2 \quad 24=3 \quad 34=1 \\
& 41=3 \quad 42=1 \quad 43=2 \\
& 15=3 \quad 25=1 \quad 35=2 \\
& 51=2 \quad 52=3 \quad 53=1 \\
& 45=0 \quad 54=0
\end{aligned}
$$

d'où

$$
\begin{array}{rlrrrr}
\boldsymbol{D}_{1}^{2}=\nabla_{1}^{2}-\nabla & \boldsymbol{D}_{2}^{2}=\nabla_{2}^{2}-\nabla & \boldsymbol{D}_{3}^{2} & =\nabla_{3}^{2}-\nabla & \boldsymbol{D}_{4}^{2}=\nabla_{4}^{2}-\nabla_{5} & \boldsymbol{D}_{5}^{2}=\nabla_{5}^{2}-\nabla_{4} \\
\boldsymbol{D}_{2} \boldsymbol{D}_{3} & =\nabla_{2} \nabla_{3}-\nabla_{4} & \boldsymbol{D}_{3} \boldsymbol{D}_{1} & =\nabla_{3} \nabla_{1}-\nabla_{4} & \boldsymbol{D}_{1} \boldsymbol{D}_{2} & =\nabla_{1} \nabla_{2}-\nabla_{4} \\
& =\nabla_{3} \nabla_{2}-\nabla_{5} & & =\nabla_{1} \nabla_{3}-\nabla_{5} & =\nabla_{2} \nabla_{1}-\nabla_{5} \\
\boldsymbol{D}_{1} \boldsymbol{D}_{4} & =\nabla_{1} \nabla_{4}-\nabla_{2} & \boldsymbol{D}_{2} \boldsymbol{D}_{4} & =\nabla_{2} \nabla_{4}-\nabla_{3} & \boldsymbol{D}_{3} \boldsymbol{D}_{4} & =\nabla_{3} \nabla_{4}-\nabla_{1} \\
& =\nabla_{4} \nabla_{1}-\nabla_{3} & & =\nabla_{4} \nabla_{2}-\nabla_{1} & & =\nabla_{4} \nabla_{3}-\nabla_{2}
\end{array}
$$

Journal für Mathematik Bd. LIX. Heft 4. 


$$
\begin{aligned}
& \boldsymbol{D}_{\mathbf{1}} \boldsymbol{D}_{\mathbf{5}}=\nabla_{1} \nabla_{5}-\nabla_{\mathbf{3}} \quad \boldsymbol{D}_{2} \boldsymbol{D}_{5}=\nabla_{2} \nabla_{5}-\nabla_{1} \quad \boldsymbol{D}_{3} \boldsymbol{D}_{5}=\nabla_{3} \nabla_{5}-\nabla_{2} \\
& =\nabla_{5} \nabla_{1}-\nabla_{2} \quad=\nabla_{5} \nabla_{2}-\nabla_{3} \quad=\nabla_{5} \nabla_{3}-\nabla_{1} \\
& D_{4} D_{5}=\nabla_{4} \nabla_{5}-\nabla \\
& =\nabla_{5} \nabla_{4}-\nabla \text {. }
\end{aligned}
$$

De là on déduit

$\left(\right.$ abcdefgh $f_{1} g_{1} h_{1} f_{2} g_{2} h_{2} k \widehat{k}\left(D_{1} D_{2} D_{3} D_{4} D_{5}\right)^{2}$

\begin{tabular}{|c|c|c|c|c|c|}
\hline $3=3$ & & & $3^{2} 2=2$ & & שי \\
\hline $1=1$ & $13=2$ & $3^{2}=1$ & $1^{2} 3=3$ & $31=2$ & $31^{2}-2$ \\
\hline $2=2$ & $21=3$ & $21^{2}=2$ & ${ }^{2} 1=1$ & $12=3$ & -1 \\
\hline & $14=1$ & $=3$ & $4=4$ & $141=5$ & $2 \quad 1$ \\
\hline & & & $4=4$ & son & \\
\hline $3=1$ & $434=3$ & & $4=4$ & $343=$ & $43^{2}=4$ \\
\hline 10 & $515=1$ & $15^{2}=2$ & $5=5$ & $151=4$ & $51^{2}=5$ \\
\hline $2=1$ & $525=2$ & $25^{2}=3$ & $5=5$ & $252=4$ & $52^{2}=5$ \\
\hline $3=2$ & $535=3$ & $=1$ & $5=5$ & $353=4$ & $3^{2}=5$ \\
\hline & & & & & \\
\hline $5=1$ & $451=1$ & & $15=2$ & 154 & $541=1$ \\
\hline $5=2$ & $452=2$ & $=1$ & $25=1$ & 35 & $542=3$ \\
\hline $5=3$ & $453=3$ & $=2$ & $5=1$ & $4=3$ & $3=3$ \\
\hline & $342=4$ & $=\mathbf{5}$ & $24=0$ & & $432=0$ \\
\hline & $143=4$ & & $x-0$ & & $413=0$ \\
\hline $4-5$ & $241=4$ & $412=5$ & $214=0$ & $142=0$ & $421=0$ \\
\hline $5-0$ & $352=0$ & $523=0$ & $325=4$ & $253=5$ & $532=4$ \\
\hline & $153=0$ & $531=0$ & $135=4$ & $351=5$ & $513=4$ \\
\hline & & $512=0$ & 015 & $152=5$ & $521 \mp 4$ \\
\hline $23=2$ & $231=3$ & $312=1$ & $213=1$ & $132=3$ & $321=2$ \\
\hline
\end{tabular}

$$
\begin{aligned}
&=\left(a b c d e f g h f_{1} g_{1} h_{1} f_{2} g_{2} h_{2} \hat{k} \widehat{(} \nabla_{1} \nabla_{2} \nabla_{3} \nabla_{4} \nabla_{5}\right)^{2} \\
&-(a+b+c+2 k) \nabla \\
&-\left(g_{1}+h_{1}+g_{2}+h_{2}\right) \nabla_{1} \\
&-\left(h_{1}+f_{1}+h_{2}+f_{2}\right) \nabla_{2} \\
&-\left(f_{1}+g_{1}+f_{2}+g_{2}\right) \nabla_{3} \\
&-(f+g+h+e) \nabla_{4} \\
&-(f+g+h+d) \nabla_{5} .
\end{aligned}
$$

Pour le troisième ordre on trouve

$$
1^{3}=1 \quad 2^{3}=2 \quad 3^{3}=3 \quad 4^{3}=0 \quad 5^{3}=0
$$


Introduisons des signes abrégés pour les coefficients de manière que le lettre qui forme la seconde partie d'une quelconque des équations ci-dessous écrites soit le coefficient de l'expression qui en forme la première partie:

$$
\begin{array}{rll}
1^{3}=a & 2^{3}=b \quad 3^{3}=c \quad 4^{3}=d \quad 5^{3}=e \\
2^{2} 3=f & 3^{2} 1=g & 1^{2} 2=b \\
23^{2}=f_{1} & 31^{2}=g_{1} & 12^{2}=h_{1} \\
1^{2} 4=l & 2^{2} 4=m & 3^{2} 4=n \\
14^{2}=l_{1} & 24^{2}=m_{1} & 34^{2}=n_{1} \\
1^{2} 5=p & 2^{2} 5=q & 3^{2} 5=r \\
15^{2}=p_{1} & 25^{2}=q_{1} & 35^{2}=r_{1} \\
& 4^{2} 5=k \quad 45^{2}=k_{1} \\
145=s & 245=t & 345=u \\
234=s_{1} & 314=t_{1} & 124=u_{1} \\
235=s_{2} & 315=t_{2} & 125=u_{2} \\
& 123=v
\end{array}
$$

on aura, après toutes reductions

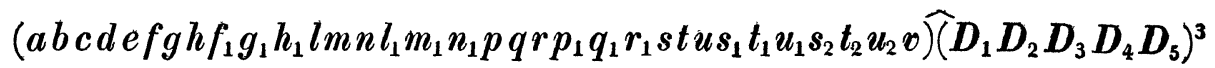

$$
\left.\begin{array}{rl}
= & a x^{3}+\left(c+d+3 u+3 u_{1}\right) y^{3}+\left(b+e+3 q+3 q_{1}\right) z^{3} \\
& +3\left(f_{1}+m_{1}+r+k+2 u+2 s_{1}\right) y^{2} z+3\left(f+m+r_{1}+k_{1}+2 t+2 s_{2}\right) y z^{2} \\
& +3\left(h_{1}+p_{1}+2 u\right) z^{2} x+3(h+p) z x^{2} \\
& +3\left(q_{1}+l\right) x^{2} y+3\left(q+l+2 t_{1}\right) x y^{2} \\
& +6\left(s+u_{1}+t_{2}+v\right) x y z \\
& +\left(f_{1}+n+q_{1}+k_{1}+2 u+2 s_{2}\right) x^{3}+\left(h_{1}+q\right) y^{3}+\left(q_{1}+u_{1}+t_{1}\right) z^{3} \\
& +\left(f+2 h+2 t+2 u+2 u_{2}\right) y^{2} z+\left(a+2 l+l_{1}+p+k+2 s+2 s_{1}+2 v\right) y z^{2} \\
& +\left(d+2 g+h+l+2 n+2 l_{1}+m_{1}+2 u+2 u_{1}+2 t_{2}\right) z^{2} x \\
& +\left(c+2 n+2 r+r_{1}+2 k+2 s+2 t+2 s_{1}+2 t_{1}+2 u_{2}+2 v\right) z x^{2} \\
& +\left(e+2 f+g+2 q+r+p_{1}+2 r_{1}+2 t+2 s_{1}+2 t_{2}\right) x^{2} y \\
& +\left(b+m+2 q_{1}+2 s_{2}+2 u_{2}+2 v\right) x y^{2} \\
& +2\left(f_{1}+g_{1}+k_{1}+q+m_{1}+p+p_{1}+k_{1}+s+u+t_{1}+u_{1}+t_{2}+t_{2}\right) x y z
\end{array}\right\}
$$


$+\left(s+t_{1}+u_{2}+v\right) x^{3}+\left(t+u_{1}+s_{2}+v\right) y^{3}+\left(u+s_{1}+t_{2}+v\right) z^{3}$

$+\left(f_{1}+g_{i}+h_{1}+l+l_{1}+q+q_{1}+k+s+u+s_{1}+t_{1}+t_{2}+u_{2}\right) y^{2} z$ $+\left(f+g+h+n+n_{1}+p+p_{1}+k_{1}+s+t+t_{1}+u_{1}+s_{2}+u_{2}\right) y z^{2}$ $+\left(f_{1}+g_{1}+h_{1}+m+m_{1}+r+r_{1}+k+s+t+t_{1}+u_{1}+s_{2}+u_{2}\right) z^{2} x$. $+\left(f+g+h+l+l_{1}+q+q_{1}+k_{1}+t+u+s_{1}+u_{1}+s_{2}+t_{2}\right) z x^{2}$ $+\left(f_{1}+g_{1}+h_{1}+n+n_{1}+p+p_{1}+k+t+u+s_{1}+u_{1}+s_{2}+t_{2}\right) x^{2} y$ $6 \frac{d^{3}}{d x d y d z}$ $+\left(f+g+h+m+m_{1}+r+r_{1}+k_{1}+s+u+s_{1}+t_{1}+t_{2}+u_{2}\right) x y^{2}$ $+\left(a+b+c+d+e+l+m+n+l_{1}+m_{1}+n_{1}+p+q+r+p_{1}+q_{1}+r_{1}\right.$ $\left.+s+t+u+s_{1}+t_{1}+u_{1}+s_{2}+t_{2}+u_{2}+v\right) x y z$

$$
=a \nabla_{1}^{3}+b \nabla_{2}^{3}+c \nabla_{3}^{3}+d \nabla_{4}^{3}+e \nabla_{5}^{3}
$$

$+f\left(\nabla_{2}^{2} \nabla_{3}+\nabla_{2} \nabla_{3} \nabla_{2}+\nabla_{3} \nabla_{2}^{2}\right)+g\left(\nabla_{3}^{2} \nabla_{1}+\nabla_{3} \nabla_{1} \nabla_{3}+\nabla_{1} \nabla_{3}^{2}\right)+h\left(\nabla_{1}^{2} \nabla_{2}+\nabla_{1} \nabla_{2} \nabla_{1}+\nabla_{1} \nabla_{2}^{2}\right)$ $+f_{1}\left(\nabla_{2} \nabla_{3}^{2}+\nabla_{3} \nabla_{2} \nabla_{3}+\nabla_{3}^{2} \nabla_{2}\right)+g_{1}\left(\nabla_{3} \nabla_{1}^{2}+\nabla_{1} \nabla_{3} \nabla_{1}+\nabla_{1}^{2} \nabla_{3}\right)+h_{1}\left(\nabla_{1} \nabla_{2}^{2}+\nabla_{2} \nabla_{1} \nabla_{2}+\nabla_{2}^{2} \nabla_{1}\right)$ $+l\left(\nabla_{1}^{2} \nabla_{4}+\nabla_{1} \nabla_{4} \nabla_{1}+\nabla_{4} \nabla_{1}^{2}\right)+m\left(\nabla_{2}^{2} \nabla_{4}+\nabla_{2} \nabla_{4} \nabla_{2}+\nabla_{4} \nabla_{2}^{2}\right)+n\left(\nabla_{3}^{2} \nabla_{4}+\nabla_{3} \nabla_{4} \nabla_{3}+\nabla_{4} \nabla_{3}^{2}\right)$ $+l_{1}\left(\nabla_{1} \nabla_{4}^{2}+\nabla_{4} \nabla_{1} \nabla_{4}+\nabla_{4}^{2} \nabla_{1}\right)+m_{1}\left(\nabla_{2} \nabla_{4}^{2}+\nabla_{4} \nabla_{2} \nabla_{4}+\nabla_{2} \nabla_{4}^{2}\right)+n_{1}\left(\nabla_{3} \nabla_{4}^{2}+\nabla_{4} \nabla_{3} \nabla_{4}+\nabla_{4}^{2} \nabla_{3}\right)$ $+\boldsymbol{p}\left(\nabla_{1}^{2} \nabla_{5}+\nabla_{1} \nabla_{5} \nabla_{1}+\nabla_{5} \nabla_{1}^{2}\right)+\boldsymbol{q}\left(\nabla_{2}^{2} \nabla_{5}+\nabla_{2} \nabla_{5} \nabla_{2}+\nabla_{5} \nabla_{2}^{2}\right)+r\left(\nabla_{3}^{2} \nabla_{5}+\nabla_{3} \nabla_{5} \nabla_{3}+\nabla_{5} \nabla_{3}^{2}\right)$ $+\boldsymbol{p}_{1}\left(\nabla_{1} \nabla_{5}^{2}+\nabla_{5} \nabla_{1} \nabla_{5}+\nabla_{5}^{2} \nabla_{1}\right)+\boldsymbol{q}_{1}\left(\nabla_{2} \nabla_{5}^{2}+\nabla_{5} \nabla_{2} \nabla_{5}+\nabla_{5}^{2} \nabla_{2}\right)+\boldsymbol{r}_{1}\left(\nabla_{3} \nabla_{5}^{2}+\nabla_{5} \nabla_{3} \nabla_{5}+\nabla_{5}^{2} \nabla_{3}\right)$ $+k\left(\nabla_{4}^{2} \nabla_{5}+\nabla_{4} \nabla_{5} \nabla_{4}+\nabla_{5} \nabla_{4}^{2}\right)+k_{1}\left(\nabla_{4} \nabla_{5}^{2}+\nabla_{5} \nabla_{4} \nabla_{5}+\nabla_{5} \nabla_{4}^{2}\right)$ $+s\left(\nabla_{1} \nabla_{4} \nabla_{5}+\nabla_{4} \nabla_{5} \nabla_{1}+\nabla_{5} \nabla_{1} \nabla_{4}+\nabla_{4} \nabla_{1} \nabla_{5}+\nabla_{1} \nabla_{5} \nabla_{4}+\nabla_{5} \nabla_{4} \nabla_{1}\right)$ $+\boldsymbol{t}\left(\nabla_{2} \nabla_{4} \nabla_{5}+\nabla_{4} \nabla_{5} \nabla_{2}+\nabla_{5} \nabla_{2} \nabla_{4}+\nabla_{4} \nabla_{2} \nabla_{5}+\nabla_{2} \nabla_{5} \nabla_{4}+\nabla_{5} \nabla_{4} \nabla_{2}\right)$ $+u\left(\nabla_{3} \nabla_{4} \nabla_{5}+\nabla_{4} \nabla_{5} \nabla_{3}+\nabla_{5} \nabla_{3} \nabla_{4}+\nabla_{4} \nabla_{3} \nabla_{5}+\nabla_{3} \nabla_{5} \nabla_{4}+\nabla_{5} \nabla_{4} \nabla_{3}\right)$ $+s_{1}\left(\nabla_{2} \nabla_{3} \nabla_{4}+\nabla_{3} \nabla_{4} \nabla_{2}+\nabla_{4} \nabla_{2} \nabla_{3}+\nabla_{3} \nabla_{2} \nabla_{4}+\nabla_{2} \nabla_{4} \nabla_{3}+\nabla_{4} \nabla_{3} \nabla_{2}\right)$ $+t_{1}\left(\nabla_{3} \nabla_{1} \nabla_{4}+\nabla_{1} \nabla_{4} \nabla_{3}+\nabla_{4} \nabla_{3} \nabla_{1}+\nabla_{1} \nabla_{3} \nabla_{4}+\nabla_{3} \nabla_{4} \nabla_{1}+\nabla_{4} \nabla_{1} \nabla_{3}\right)$ $+u_{1}\left(\nabla_{1} \nabla_{2} \nabla_{4}+\nabla_{2} \nabla_{4} \nabla_{1}+\nabla_{4} \nabla_{1} \nabla_{2}+\nabla_{2} \nabla_{1} \nabla_{4}+\nabla_{1} \nabla_{4} \nabla_{2}+\nabla_{4} \nabla_{2} \nabla_{1}\right)$ $+s_{2}\left(\nabla_{2} \nabla_{3} \nabla_{5}+\nabla_{3} \nabla_{5} \nabla_{2}+\nabla_{5} \nabla_{2} \nabla_{3}+\dot{\nabla}_{3} \nabla_{2} \nabla_{5}+\nabla_{2} \nabla_{5} \nabla_{3}+\nabla_{5} \nabla_{3} \nabla_{2}\right)$ $+t_{2}\left(\nabla_{3} \nabla_{1} \nabla_{5}+\nabla_{1} \nabla_{5} \nabla_{3}+\nabla_{5} \nabla_{3} \nabla_{1}+\nabla_{1} \nabla_{3} \nabla_{5}+\nabla_{3} \nabla_{5} \nabla_{1}+\nabla_{5} \nabla_{1} \nabla_{3}\right)$ $+u_{2}\left(\nabla_{1} \nabla_{2} \nabla_{5}+\nabla_{2} \nabla_{5} \nabla_{1}+\nabla_{5} \nabla_{1} \nabla_{2}+\nabla_{2} \nabla_{1} \nabla_{5}+\nabla_{1} \nabla_{5} \nabla_{2}+\nabla_{5} \nabla_{2} \nabla_{1}\right)$ $+v\left(\nabla_{1} \nabla_{2} \nabla_{3}+\nabla_{2} \nabla_{3} \nabla_{1}+\nabla_{3} \nabla_{1} \nabla_{2}+\nabla_{2} \nabla_{1} \nabla_{3}+\nabla_{1} \nabla_{3} \nabla_{2}+\nabla_{3} \nabla_{2} \nabla_{1}\right)$

$-3\left(t_{1}+u_{1}+t_{2}+u_{2}\right) \nabla_{1}^{2}-3\left(u_{1}+s_{1}+u_{2}+s_{2}\right) \nabla_{2}^{2}-3\left(s_{1}+t_{1}+s_{2}+t_{2}\right) \nabla_{3}^{2}-3\left(s_{1}+t_{1}+u_{1}\right) \nabla_{4}^{2}$ $-3\left(s_{2}+t_{2}+u_{2}\right) \nabla_{5}^{2}$

$-3\left(a+g+h_{1}+2 s\right) \nabla \nabla_{1}-3\left(b+h+f_{1}+2 t\right) \nabla \nabla_{2}-3\left(c+f+g_{1}+2 u\right) \nabla \nabla_{3}$ $-3(l+m+n) \nabla \nabla_{4}-3(p+q+r) \nabla \nabla_{5}$

$-\left(2 m+2 q+n+r+t_{1}+2 u_{1}+t_{2}+2 u_{2}\right) \nabla_{2} \nabla_{3}-\left(m+q+2 n+2 r+2 t_{1}+u_{1}+2 t_{2}+u_{2}\right) \nabla_{3} \nabla_{2}$ $-\left(l+p+2 n+2 r+2 s_{1}+u_{1}+2 s_{2}+u_{2}\right) \nabla_{3} \nabla_{1}-\left(2 l+2 p+n+r+s_{1}+2 u_{1}+s_{2}+2 u_{2}\right) \nabla_{1} \nabla_{3}$ 
$-\left(2 l+2 p+m+q+s_{1}+2 t_{1}+s_{2}+2 t_{2}\right) \nabla_{1} \nabla_{2}-\left(l+p+2 m+2 q+2 s_{1}+t_{1}+2 s_{2}+t_{2}\right) \nabla_{2} \nabla_{1}$ $-\left(2 h+2 p_{1}+2 g_{1}+m_{1}+n_{1}+t+u+2 v\right) \nabla_{1} \nabla_{4}-\left(h+p_{1}+2 m_{1}+g_{1}+2 n_{1}+2 t+2 u+v\right) \nabla_{4} \nabla_{1}$ $-\left(2 f+2 h_{1}+l_{1}+2 q_{1}+n_{1}+u+s+2 v\right) \nabla_{2} \nabla_{4}-\left(f+h_{1}+2 l_{1}+q_{1}+2 n_{1}+2 u+2 s+v\right) \nabla_{4} \nabla_{2}$ $-\left(2 f_{1}+l_{1}+m_{1}+2 r_{1}+2 v+s+t+2 g\right) \nabla_{3} \nabla_{4}-\left(f_{1}+2 l_{1}+2 m_{1}+r_{1}+v+2 s+2 t+g\right) \nabla_{4} \nabla_{3}$ $-\left(2 g_{1}+2 h+2 l_{1}+q_{1}+r_{1}+t+u+2 v\right) \nabla_{1} \nabla_{5}-\left(g_{1}+h+l_{1}+2 q_{1}+2 r_{1}+2 t+2 u+v\right) \nabla_{5} \nabla_{1}$ $-\left(2 f+2 h_{1}+p_{1}+2 m_{1}+r_{1}+u+s+2 v\right) \nabla_{2} \nabla_{5}-\left(f+h_{1}+2 p_{1}+m_{1}+2 r_{1}+2 u+2 s+v\right) \nabla_{5} \nabla_{2}$ $-\left(2 g+p_{1}+q_{1}+2 n_{1}+2 f_{1}+s+t+2 v\right) \nabla_{3} \nabla_{3}-\left(g+2 p_{1}+q_{1}+n_{1}+f_{1}+2 s+2 t+v\right) \nabla_{5} \nabla_{3}$

$$
-3\left(d+e+s_{1}+t_{1}+u_{1}+s_{2}+t_{2}+u_{2}\right) \nabla_{4} \nabla_{5}
$$

$+2\left(d+e+3 s_{1}+3 t_{1}+3 u_{i}+3 s_{2}+3 t_{2}+3 u_{2}\right) \nabla$

$+2\left(a+f+f_{1}+2 g+2 h_{1}+l+m+n+p+q+r+4 s+t+u+2 v\right) \nabla_{1}$

$+2\left(b+2 f_{1}+g+g_{1}+2 h+l+m+n+p+q+r+s+4 t+u+2 v\right) \nabla_{2}$

$+2\left(c+2 f+2 g_{1}+h+h_{1}+l+m+n+p+q+r+s+t+4 u+2 v\right) \nabla_{3}$

$+2\left(2 l_{1}+2 m_{1}+2 n_{1}+p_{1}+q_{1}+r_{1}+s_{1}+t_{1}+u_{1}+2 s_{2}+2 t_{2}+2 u_{2}\right) \nabla_{4}$

$+2\left(l_{1}+m_{1}+n_{1}+2 p_{1}+2 q_{1}+2 r_{1}+2 s_{1}+2 t_{1}+2 u_{1}+s_{2}+t_{2}+u_{2}\right) \nabla_{5}$.

Londres, 1861. 